\title{
Artemisinin compounds sensitize cancer cells to ferroptosis by regulating iron homeostasis
}

\author{
Guo-Qing Chen ${ }^{1,2} \cdot$ Fahad A. Benthani ${ }^{2} \cdot$ Jiao $\mathrm{Wu}^{2,3} \cdot$ Deguang Liang ${ }^{2} \cdot{\text { Zhao-Xiang Bian } \mathbb{D}^{1} \cdot \text { Xuejun Jiang }}^{2}$
}

Received: 13 March 2019 / Revised: 24 April 2019 / Accepted: 2 May 2019 / Published online: 21 May 2019

(c) ADMC Associazione Differenziamento e Morte Cellulare 2019

\begin{abstract}
The antimalarial drug artemisinin and its derivatives have been explored as potential anticancer agents, but their underlying mechanisms are controversial. In this study, we found that artemisinin compounds can sensitize cancer cells to ferroptosis, a new form of programmed cell death driven by iron-dependent lipid peroxidation. Mechanistically, dihydroartemisinin (DAT) can induce lysosomal degradation of ferritin in an autophagy-independent manner, increasing the cellular free iron level and causing cells to become more sensitive to ferroptosis. Further, by associating with cellular free iron and thus stimulating the binding of iron-regulatory proteins (IRPs) with mRNA molecules containing iron-responsive element (IRE) sequences, DAT impinges on IRP/IRE-controlled iron homeostasis to further increase cellular free iron. Importantly, in both in vitro and a mouse xenograft model in which ferroptosis was triggered in cancer cells by the inducible knockout of GPX4, we found that DAT can augment GPX4 inhibition-induced ferroptosis in a cohort of cancer cells that are otherwise highly resistant to ferroptosis. Collectively, artemisinin compounds can sensitize cells to ferroptosis by regulating cellular iron homeostasis. Our findings can be exploited clinically to enhance the effect of future ferroptosis-inducing cancer therapies.
\end{abstract}

\section{Introduction}

Programmed cell death (PCD) plays an important role in normal biology, and its dysregulation contributes to the development of various diseases [1-3]. Recent studies have

These authors contributed equally: Guo-Qing Chen, Fahad A. Benthani

Edited by H.-U. Simon

Supplementary information The online version of this article (https:// doi.org/10.1038/s41418-019-0352-3) contains supplementary material, which is available to authorized users.

Zhao-Xiang Bian

bzxiang@hkbu.edu.hk

Xuejun Jiang

jiangx@mskcc.org

1 School of Chinese Medicine, Hong Kong Baptist University, Kowloon Tong, Hong Kong, China

2 Cell Biology Program, Memorial Sloan Kettering Cancer Center, New York City, NY 10065, USA

3 National Translational Science Center for Molecular Medicine, Department of Cell Biology, School of Basic Medicine, Air Force Medical University, Xi' an 710032, China shown that in addition to apoptosis, the best-characterized form of PCD, there are various other forms, including ferroptosis, an iron-dependent (thus the name) modality of cell death driven by lipid peroxidation [4-6]. Although the precise mechanisms and physiological function of ferroptosis remain elusive, mounting evidence indicates that ferroptosis is involved in multiple pathological conditions, including cancer [6-9]. Several studies further suggested that ferroptosis may be a native tumor suppressive mechanism, contributing to the antitumor function of p53, BAP1, and fumarase, all clinically relevant tumor suppressors [10-12]. Strikingly, recent studies showed that metastasis-prone mesenchymal cancer cells are particularly sensitive to ferroptosis triggered by both cysteine deprivation and inhibition of lipid peroxide-clearance enzyme, glutathione peroxidase-4 (GPX4), although these cancer cells are often resistant to chemo and targeted therapies [13]. Therefore, induction of ferroptosis in cancer cells holds the promise to be a novel therapeutic approach.

Artemisinin (ART), a natural product isolated from the traditional Chinese plant Artemisia аппиа, and its semisynthetic derivatives with improved bioavailability are effective antimalarial drugs $[14,15]$. While the precise mechanism of action of ART and its derivatives in malarial treatment is debatable $[16,17]$, the consensus is that the endoperoxide 
bridge within these compounds is essential for their therapeutic effect [18-20]. The endoperoxide bond is highly reactive, and it can react with biological sources of iron within malaria-infected erythrocytes [20]. This reaction generates carbon-centered free radicals and reactive oxygen species (ROS), contributing to the killing of the parasites [21].

Interestingly, ART and its derivatives have also been implicated in the treatment of other diseases, including cancer [22-24]. The anticancer effect of these compounds has been attributed to their ability to induce various cellular processes, ranging from DNA damage response, the lysosome-mediated catabolic process macroautophagy (autophagy hereafter) and oxidative stress, to diverse modes of cell death including apoptosis and ferroptosis [25-29]. Adding to the obscurity of the anticancer mechanism, the cytotoxicity of these compounds against cancer cells is often low (in most cases with EC50 > 100 $\mu \mathrm{M}$ [30-32]).

Because ART and its derivatives can react with iron and trigger ROS production in cells, we sought to investigate in this study their potential role in regulating ferroptosis in cancer cells. We found that ART compounds, including dihydroartemisinin (DAT), an ART derivative with high bioavailability, can enhance the sensitivity of cancer cells to ferroptosis induced by both cysteine deprivation and GPX4 inhibition in a lysosome-dependent, but autophagyindependent manner. Further, DAT can modulate cellular iron homeostasis regulated by the iron-storage protein ferritin and the IRP-IRE signaling route [33], thus impacting ferroptosis. Importantly, in both cellular and mouse xenograft models, we demonstrate that DAT can render ferroptosis-resistant cancer cells more sensitive to ferroptotic death, suggesting an effective combinatory cancer therapy involving DAT and ferroptosis induction.

\section{Materials and methods}

\section{Antibodies and reagents}

Primary antibodies used were anti-LC3B (L7543, SigmaAldrich, St. Louis, MO, USA), anti-p62 (23214, Cell Signaling, Danvers, MA, USA), anti-NCOA4 (A302-272A, Bethyl Laboratories, Montgomery TX, USA), anti-FTH1 (4393S, Cell Signaling), anti-ATG5 (A0731, SigmaAldrich), anti-GPX4 (ab125066, Abcam, Cambridge UK), anti-Cas9 (19526S, Cell Signaling), anti-Actin (A5316, Sigma-Aldrich), anti-LAMP2A (ab18528, Abcam) and antiKi67 (9449, Cell Signaling). The compounds used are dihydroartemisinin (D7439, Sigma-Aldrich), artemisinin (361593, Sigma-Aldrich), artesunate (A3731, SigmaAldrich), artemether (A9361, Sigma-Aldrich), erastin (E7781, Sigma-Aldrich), bafilomycin A1 (B1793, SigmaAldrich), chloroquine (C6628, Sigma-Aldrich), RSL3
(S8155, Selleckchem, Houston, TX USA), Liproxstatin-1 (SML1414, Sigma-Aldrich), SYTOX Green (S7020, Thermo Fisher, Waltham, MA, USA), propidium iodide (556463, BD Biosciences, San Jose, CA, USA) and FIN56 (25180, Cayman Chemical, Ann Arbor, MI, USA).

\section{Cell culture}

NCI-H292, HCT116, HT29, SW480, MDA-MB-453, and MCF7 cell lines were obtained from the American Tissue Culture Collection (ATCC) and cultured in media conditions recommended by the ATCC. MEF and HT1080 cell lines were cultured in high glucose DMEM supplemented with $10 \%$ (v/v) FBS, glutamine $(2 \mathrm{mM})$, penicillin (100 I. $\mathrm{U} . / \mathrm{ml})$ and streptomycin $(0.1 \mathrm{mg} / \mathrm{ml})$ in a humidified atmosphere containing $5 \% \mathrm{CO}_{2}$ at $37^{\circ} \mathrm{C}$. All cell lines were tested for mycoplasma prior to the commencement of experiments. Unless otherwise indicated, cell culture medium was changed every three days, and cells were passaged using $0.05 \%$ trypsin/EDTA.

\section{Cell death quantification}

All cell death experiments were conducted in $~ 70-80 \%$ confluent wells of 6-well plates. For cysteine starvation experiments, cells were washed twice with PBS and cultured in cystine free medium in the presence of $10 \%(\mathrm{v} / \mathrm{v})$ dialyzed FBS for the indicated times. Cells were treated with the ferroptosis inducing compounds erastin and RSL3 and the ferroptosis inhibitor Ferrostatin-1 as indicated. To quantify cell death, $1 \mu \mathrm{g} / \mathrm{ml}$ of PI or $1 \mu \mathrm{M}$ SYTOX Green was added to the culture media and the cells were analyzed by flow cytometry for PI + or Sytox + cells.

\section{Measurement of glutathione activity}

Cells were treated as indicated, and cellular GSH level was assessed using the Quantichrom Glutathione Assay Kit (DIGT-250, BioAssay Systems, Hayward, CA, USA) according to the manufacturer's instructions.

\section{Measurement of lipid reactive oxidative species}

Cells were treated as indicated and were then incubated for $1 \mathrm{~h}$ with $50 \mu \mathrm{M}$ of lipid peroxidation sensor BODIPY 581/ 591 C11 (Thermo Fisher, Cat \#D3861). Excess BODIPY C11 was removed by washing the cells twice with PBS. Labeled cells were trypsinized and resuspended in PBS and $5 \%(\mathrm{v} / \mathrm{v})$ FBS. Oxidation of the polyunsaturated butadienyl portion of BODIPY $\mathrm{C} 11$ resulted in a shift of the fluorescence emission peak from $\sim 590 \mathrm{~nm}$ to $\sim 510 \mathrm{~nm}$ proportional to lipid ROS generation and was analyzed using a flow cytometer. 


\section{Measurement of labile iron protein}

Labile iron pool (LIP) was measured as previously described [34]. Briefly, trypsinized cells were washed twice with $0.5 \mathrm{ml}$ of PBS and incubated with $0.05 \mu \mathrm{M}$ calceinacetoxymethyl ester (AnaSpec, Fremont, CA USA) for 15 $\min$ at $37^{\circ} \mathrm{C}$. Cells were then washed twice with $0.5 \mathrm{ml}$ of PBS and incubated either with deferiprone $(100 \mu \mathrm{M})$ for $1 \mathrm{~h}$ at $37^{\circ} \mathrm{C}$ or left untreated. Cells were analyzed using a flow cytometer. Calcein was excited at $488 \mathrm{~nm}$, and fluorescence was measured at $525 \mathrm{~nm}$. The difference in the mean cellular fluorescence with and without deferiprone incubation reflects the amount of LIP.

\section{Measurement of lipid peroxidation in vitro}

Lipid peroxidation in vitro was measured according to a modified spectrophotometric detection of the products resulting from the reaction with thiobarbituric acid (TBARS), of which the most significant is malondialdehyde (MDA), one of the final products of polyunsaturated fatty acids peroxidation in the cells $[35,36]$. Briefly, $350 \mu \mathrm{M}$ arachidonic acid was dispersed in $\mathrm{H}_{2} \mathrm{O}$ with $0.02 \%$ Triton$\mathrm{X} 100.5 \mu \mathrm{M} \mathrm{FeSO} 4,500 \mu \mathrm{M}$ ascorbic acid and $10 \mu \mathrm{M}$ DAT were then added and the test samples were incubated at $37^{\circ} \mathrm{C}$ for different times. Subsequently, $0.5 \%$ thiobarbituric acid in $20 \%$ trichloroacetic acid were added to all test samples, and all samples were incubated at $95^{\circ} \mathrm{C}$ for $15 \mathrm{~min}$. The amount of TBARS produced was measured by the spectrophotometric absorbance of the supernatant at $532 \mathrm{~nm}$ using a spectrophotometer (C-5320001, Perkin Elmer, Waltham, MA USA).

\section{Inducible CRISPR/Cas9-mediated GPX4 knockout}

The lentiviral doxycycline (DOX)-inducible pCW-Cas9 vector and $\mathrm{pLX}$-sgRNA were gifts from Eric Lander and David Sabatini (\#50661 and 50662 respectively, Addgene, Watertown, MA, USA) for inducible gene knockout (iKO). The guide RNA sequence CACGCCCGATACG CTGAGTG targeting human GPX4 was used. To construct the lentiviral sgRNA vector for GPX4, a pair of oligonucleotides (forward and reverse) were annealed, phosphorylated and ligated into pLX-sgRNA. Lentiviral particles containing the sgRNA or Cas9 vectors were produced by co-transfecting the sgRNA vector with pCMV-dR8.2 dvpr (\#8455, Addgene) and pCMV-VSV-g (\#8454, Addgene) lentiviral packaging plasmids into $293 \mathrm{~T}$ cells using polyethylenimine (PEI) transfection reagent. Media was changed $12 \mathrm{~h}$ after transfection, and the virus-containing supernatant was collected and filtered $48 \mathrm{~h}$ after transfection. H292 cells in 6-well tissue culture plates were infected with pCW-Cas9 viral supernatant containing $4 \mu \mathrm{g} / \mathrm{mL}$ polybrene. Twenty-four hours after infection, the virus was removed, and cells were selected with $2 \mu \mathrm{g} / \mathrm{ml}$ puromycin. Single clones were screened for DOX-inducible Cas9 expression. $2 \mu \mathrm{g} / \mathrm{ml}$ DOX was added to the culture media for 3 days. Single clones with Cas9 expression were infected with the GPX4 gRNA virus-containing supernatant containing $8 \mu \mathrm{g} / \mathrm{ml}$ polybrene. Twenty-four hours after infection, the virus was removed, and cells were selected with $10 \mu \mathrm{g} / \mathrm{ml}$ blasticidin. Single clones with DOXinducible Cas9 expression and GPX4 knockout were amplified and used.

\section{In vivo xenograft studies}

GPX4 iKO H292 cells were inoculated by injecting $3 \times$ $10^{6}$ cells in $0.1 \mathrm{~mL}$ PBS subcutaneously in the right flank of six- to eight-week-old female athymic nude Foxn $1^{\text {nu}}$, Foxn $^{+}$mice (Envigo, East Millstone, NJ, USA). Following inoculation, the mice were monitored until they have fully recovered and are moving. Mice were randomly allocated into their respective groups (non-blinded). Tumor growth was monitored regularly via external caliper measurements. When tumors reached a mean volume of $100 \mathrm{~mm}^{3}$, mice were divided randomly into five groups: (1) vehicle group (daily i.p. saline and normal diet), (2) DAT group (daily i.p. $5 \mathrm{mg} / \mathrm{kg}$ of DAT and normal diet), (3) Dox group (daily i.p. saline and DOX food), (4) Dox + DAT group (daily i.p. $5 \mathrm{mg} / \mathrm{kg}$ of DAT and DOX diet), (5) Dox + DAT + Lip-1 group (daily i.p. $5 \mathrm{mg} / \mathrm{kg}$ of DAT and $10 \mathrm{mg} / \mathrm{kg}$ Liproxstatin- 1 and DOX diet). The tumors were measured with calipers every day, and tumor volumes were calculated using the following formula: $a^{2} \times b \times 0.4$, where " $a$ " is the smallest diameter and " $b$ " is the diameter perpendicular to " $a$ ". Tumor growth was monitored over time. Two weeks after administration, the mice were euthanized, and the tumor xenografts were immediately dissected, weighed, fixed with formalin, processed, embedded in paraffin and sectioned for further immunohistochemical analysis.

\section{Immunohistochemistry}

Antigen retrieval was performed with the Retrievagen A antigen retrieval system (550524, BD Biosciences) according to the manufacturer's instructions. Immunohistochemical staining was performed on $5 \mu \mathrm{m}$-thick paraffinembedded sections using rabbit anti-GPX4 (Abcam) and mouse anti-Ki-67 antibodies with a standard avidin-biotin HRP detection system according to the instructions of the manufacturer (anti-mouse/rabbit HRP-DAB Cell \& Tissue Staining Kit, R\&D Systems Minneapolis, MN). Tissues were counterstained with haematoxylin, dehydrated, and mounted. 


\section{Iron reporters}

We used PCR (forward primer GGATGATCACGTTA CATAACTTACGGTAA and reverse primer GCGGAAT TCCTACACATTGATCCTAGCAG) to amplify CMVIRE-YFP fragment from 5 -IRE-YFP plasmid (a gift from Dr. Jonathan Barasch [37]) and inserted this fragment into pBabe-IRES-GFP plasmid (a deposit in our lab) between the BamHI and EcoRI sites to make an intermediate 5'IREYFP-IRES-GFP reporter. To make the 5'IRE-mCherryIRES-GFP reporter or the 5'-IRE-Luc-IRES-GFP reporter, the YFP sequence was further replaced with the mCherry sequence or firefly luciferase between the AgeI and EcoRI sites.

\section{Statistical analysis}

$t$-tests were performed using Prism software (GraphPad, La Jolla, CA, USA). The Australian National Statistical Service Sample Calculator was used for sample size and power calculations. All error bars represent standard deviation ( $n$ $=3$ ) unless otherwise indicated. The $P$-value level set for statistical significance is $P \leq 0.05$.

\section{Study approval}

All animal work was conducted at Memorial SloanKettering Cancer Center in accordance with the protocol approved by the Institutional Animal Care and Use Committee (IACUC protocol 10-10-023).

\section{Results}

\section{Artemisinin and its derivatives sensitize cells to ferroptosis}

The reactivity of the endoperoxide bond in ART compounds with biological iron prompted us to investigate whether these compounds can regulate ferroptosis, an irondependent modality of cell death. In both mouse embryonic fibroblasts (MEFs) and human osteosarcoma HT1080 cells, we found that multiple ART compounds (Fig. 1a), at concentrations up to $50 \mu \mathrm{M}$, induced minimal cell death alone (data not shown). Interesting, all ART compounds sensitized cells to cysteine starvation (STV)-induced ferroptosis, in a time- and dose-dependent manner (Fig. 1b, c). Among these four ART compounds, DAT, the active metabolite of all ART compounds, was the most effective at sensitizing cells to STV-induced ferroptosis, likely due to its better bioavailability [15]. DAT was therefore used for our further investigations. In addition to STV, three pharmacological inducers of ferroptosis representing the three types of ferroptosis-inducing agents (FINs), cystine transport inhibitor erastin, GPX4 inhibitor RSL3 [4, 38], and promoter of GPX4 degradation FIN56 were also examined. Like STV, DAT also improved the sensitivity of these three inducers (Fig. 1d, S1, S2, and S3). Notably, under all these conditions, cell death was suppressed by the ferroptosis inhibitor Ferrostatin-1 [4] (Fer-1) (Fig. 1d, S1, S2, and S3). Furthermore, for erastin and RSL3, the intensity of cell death correlated with lipid peroxide generation (Fig. 1e), confirming the ferroptotic nature of the observed cell death.

Subsequently, we investigated the molecular basis underlying ferroptosis sensitization by DAT.

\section{DAT increases cellular free iron contents but has no effect on cellular glutathione}

Ferroptosis is an iron-dependent cell death process driven by lipid peroxidation [4]. Therefore, we first examined whether DAT treatment would alter the level of cellular glutathione (GSH), the primary antioxidant in cells, and that of cellular iron. Erastin induced a significant decrease of cellular GSH level (Fig. 2a), due to the inhibition of the import of cystine, which once inside the cell is reduced to cysteine, a building block for GSH synthesis. However, DAT had no effect on cellular GSH levels by itself or in the presence of erastin (Fig. 2a). On the other hand, both alone and in combination with erastin, DAT led to an increase of free iron contents in cells, quantified as cellular LIP (Fig. 2b). As expected, Deferoxamine (DFO), an iron chelator, completely suppressed ferroptosis induced by erastin alone or in combination with DAT (Fig. 2c and S4). These results indicate that the increase of cellular labile iron contents, but not cellular glutathione levels contribute to the effect of DAT on ferroptosis sensitization.

\section{The effect of DAT on ferroptosis is independent of autophagy}

It was previously reported that autophagy promotes ferroptosis by degrading cellular iron storage protein ferritin and thus increasing cellular labile iron contents [39, 40]. Interestingly, ART has been shown to stimulate autophagy, although at concentrations much higher than what was used in our study [27, 41]. Based on these previous reports and our finding that DAT can sensitize cells to ferroptosis and increase cellular labile iron contents (Figs. 1 and 2), we sought to examine whether DAT sensitizes cells to ferroptosis by stimulating autophagy. Consistent with previous reports [40], we observed that erastin treatment enhanced autophagy flux in both MEFs and HT1080 cells, i.e., it caused an increase of LC3-II production, the degradation of autophagy substrate p62 as assayed by western blot, and an increase of GFP-LC3-II puncta formation as assayed by 
A

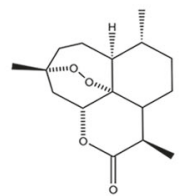<smiles>CC1CCC2C(C)C(C)CC3CC(C)C(C)C(C1)C32C</smiles>

Artemisinin

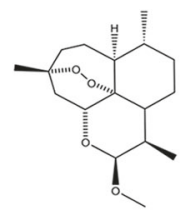

Artemether

C

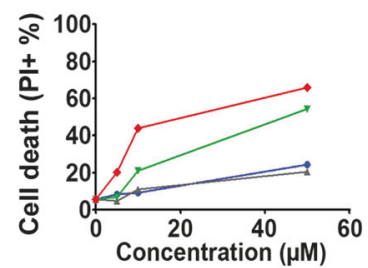

MEFs
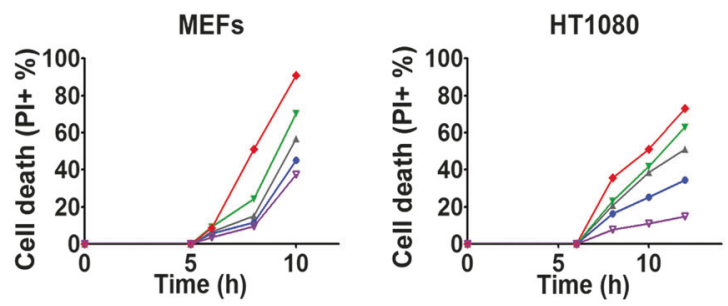

B

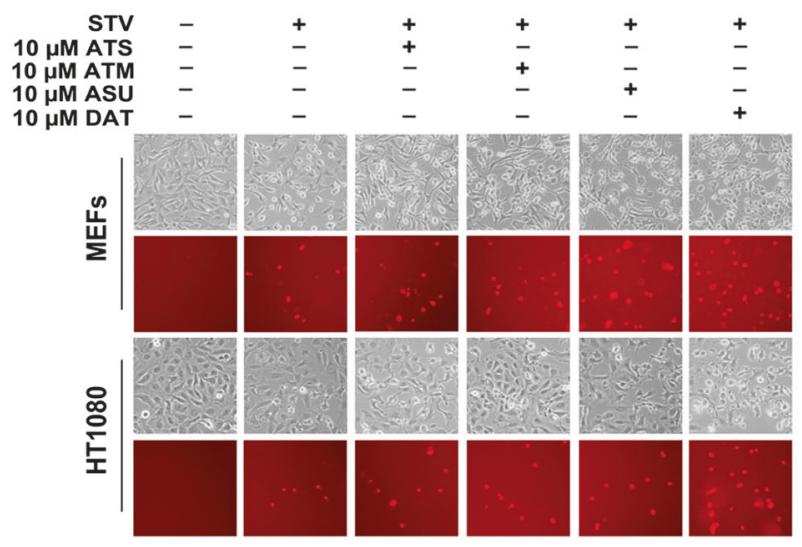

D

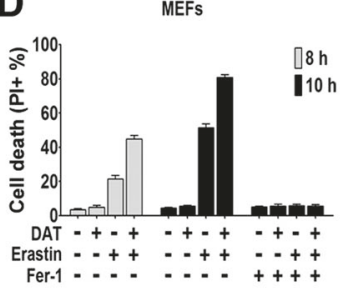

MEFs

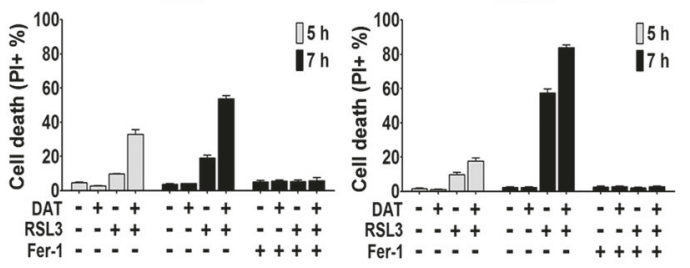

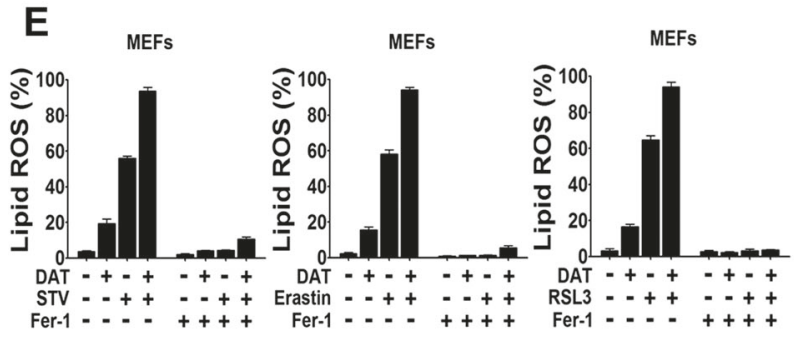

Fig. 1 Artemisinin and its derivatives enhance the sensitivity of cells to ferroptosis. a Chemical structure of four artemisinin compounds. b, $\mathbf{c}$ In b, ferroptosis was induced in MEF and HT1080 cells by cystine starvation for 8 and $10 \mathrm{~h}$ respectively with or without the indicated concentrations of ART compounds. Upper panel shows phase-contrast images and the lower panel shows propidium iodide (PI) staining to indicate dead cells at $\times 10$ magnification. $\mathbf{c}$ Flow cytometric quantification of PI positive ferroptotic MEF and HT1080 cells treated with

immunofluorescence. Lysosomal inhibitor bafilomycin A1 (bafA1) further increased LC3-II production and GFP-LC3 puncta formation but blocked p62 degradation (Fig. 3a, b). However, at a $10-\mu \mathrm{M}$ concentration, DAT did not cause an increase of autophagy flux, either used alone or in combination with erastin (Fig. 3a, b), although at this

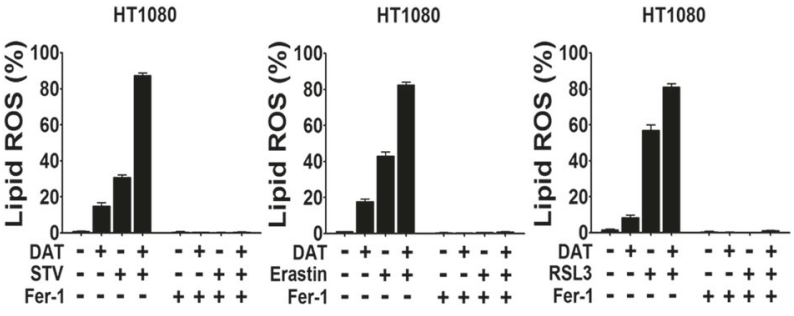

cystine starvation media for 6 and $8 \mathrm{~h}$ respectively (upper panel) or with $10 \mu \mathrm{M}$ of artemisinin compounds (lower panel). d PI positive cells were quantified by flow cytometry after treatment with erastin or RSL3 for the indicated times. e Ferrostatin blocked DAT-sensitized lipid ROS, quantified using BODIPY-C11 lipid probe using flow cytometry in MEF and HT1080 cells. For d and e, cells were treated with $10 \mu \mathrm{M}$ DAT, $1 \mu \mathrm{M}$ erastin, $0.5 \mu \mathrm{M}$ RSL3, and $1 \mu \mathrm{M}$ Ferrostatin-1. Error bars indicate standard deviation $(n=3)$

concentration it sensitized cells to ferroptosis (Fig. 1), suggesting DAT enhances the sensitivity of cells to ferroptosis in an autophagy-independent manner.

To further confirm the autophagy-independent role of DAT in ferroptosis, we used autophagy-deficient MEFs, which had the autophagy-essential gene Atg5 genetically 

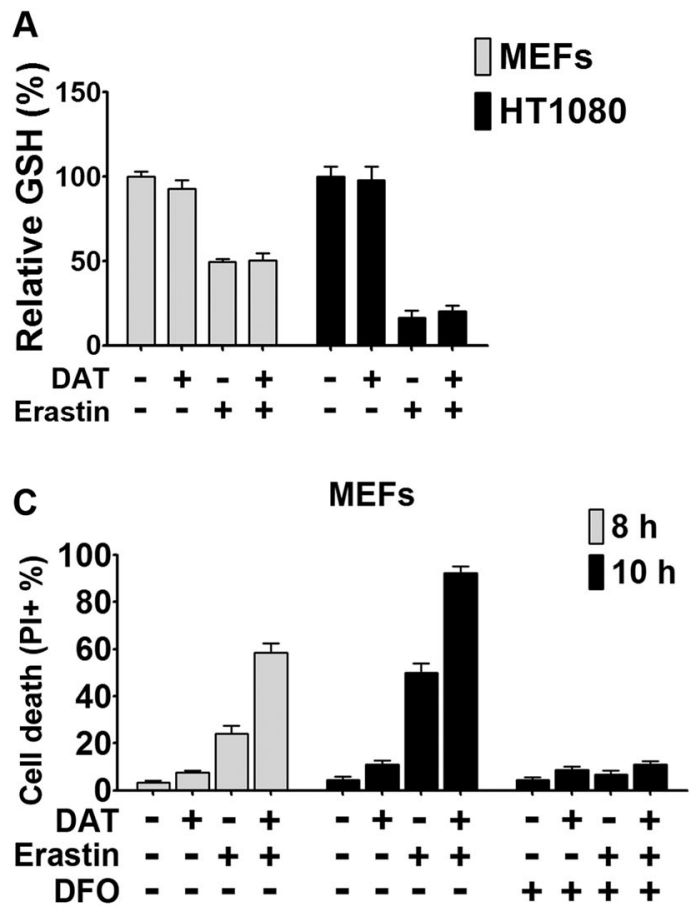

Fig. 2 DAT increases cellular free iron contents but has no effect on cellular glutathione. a Quantification of the reduced cellular GSH levels using the DTNB method by measuring optical density at $412 \mathrm{~nm}$ after erastin-induced ferroptosis with and without DAT. Values were plotted as a percentage relative to the untreated control sample. b Quantification of cellular LIP levels using the calcein-AM (C-AM)
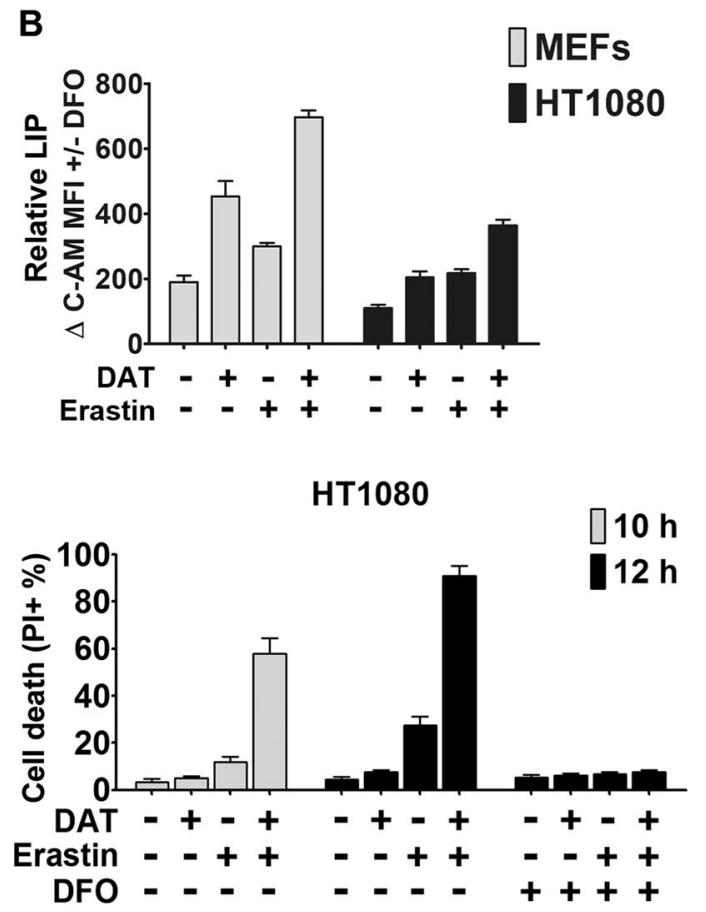

method by measuring spectrophotometric absorbance at $525 \mathrm{~nm}$. The mean fluorescence intensity (MFI) of C-AM is subtracted from the MFI of C-AM treated with DFO. c Cell death was measured by quantifying the percentage PI positive ferroptotic MEF and HT1080 cells by flow cytometry. For $\mathbf{a}-\mathbf{c}, 10 \mu \mathrm{M}$ of DAT, $1 \mu \mathrm{M}$ of erastin and $80 \mu \mathrm{M}$ of DFO concentrations were used for the indicated times
A

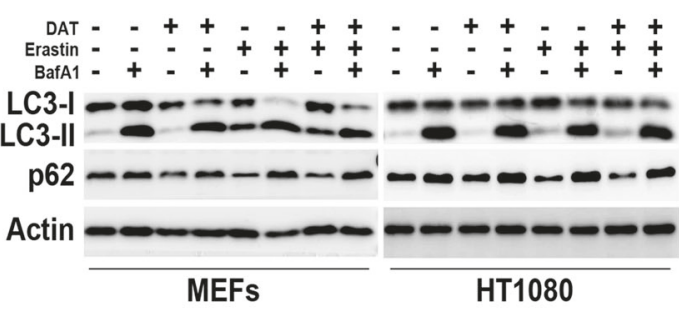

C

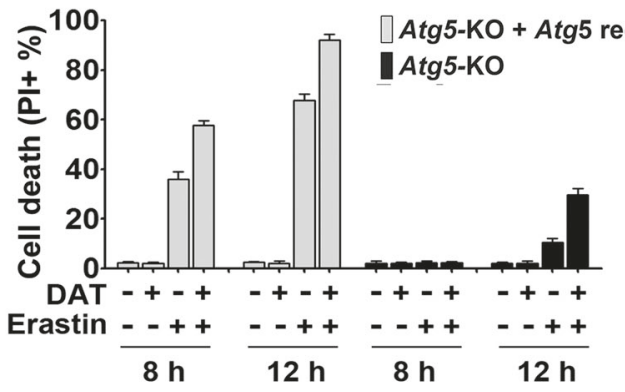

Fig. 3 DAT does not affect autophagic flux and sensitizes cells to ferroptosis through a mechanism independent of autophagy. a Ferroptosis was induced by erastin with or without the addition of DAT and BafA1. Autophagic activity was assessed by western blot analysis of LC3-I/LC3-II and p62 protein expression. b Immunofluorescence analysis of GFP-LC3-II accumulation after erastin-induced ferroptosis

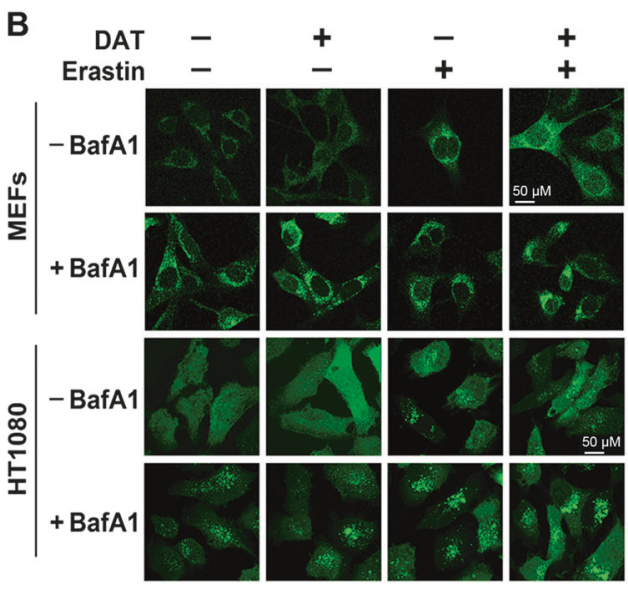

with or without BafA1. The presence of puncta indicates LC3-II. For $\mathbf{a}-\mathbf{b}$, MEFs were treated with erastin for $8 \mathrm{~h}$ and HT1080 for $10 \mathrm{~h}$. c Percentage cell death was quantified by flow cytometric analysis of PI-positive cells in Atg5-KO MEFs, or isogenic Atg5-KO cells reconstituted with ectopic Atg 5 expression. For a-c, cells were treated with $10 \mu \mathrm{M}$ DAT, $1 \mu \mathrm{M}$ erastin and $20 \mathrm{nM}$ BafA1 
knocked out (Atg5-KO). Consistent with the previous reports [40], although ferroptosis is mostly delayed in Atg5KO MEFs compared to Atg5-KO MEFs reconstituted with ectopic expression of Atg5, we found that DAT sensitized cells to erastin-induced ferroptosis in both Atg5-KO MEF cell line and its isogenic wild-type pair (Fig. 3c and S5). Under all these conditions, cell death was blocked by Ferrostatin-1 (Fig. S6). Taken together, DAT sensitizes cells to ferroptosis in an autophagy-independent manner.

\section{Lysosomal activity is required for DAT-sensitized ferroptosis}

Consistent with the autophagy-independent role of DAT in ferroptosis, DAT increased LIP in autophagy-defective MEFs (Atg5-KO) whereas erastin only increased LIP in autophagy-competent MEFs (i.e., Atg5-KO reconstituted with wild-type Atg5) (Fig. 4a). Interestingly, under both autophagy-competent and autophagy-defective conditions, the lysosomal inhibitor BafA1 blocked the increase of LIP triggered by erastin and DAT (Fig. 4a). Further, in both autophagy-competent and autophagy-defective MEFs, BafA1 inhibited ferroptosis induced by erastin alone or in combination with DAT (Fig. 4b).

Ferritin is an iron-storage protein actively involved in the regulation of cellular iron homeostasis [42]. When cells are under iron-depletion conditions (i.e., low LIP), ferritin is degraded in the lysosome to release iron to restore LIP. It has been shown that the lysosome can degrade ferritin via both autophagy-dependent (ferritinophagy) and autophagyindependent mechanisms [43-45]. We examined the effect of erastin and DAT, either alone or in combination, on the cellular levels of ferritin and its autophagy cargo receptor Ncoa4 [46]. In autophagy-competent MEFs, erastin stimulated lysosomal degradation of Ncoa4 level, which was blocked by BafA1, whereas the cellular level of ferritin was not significantly altered by erastin (Fig. 4c). This observation is consistent with a previous report showing that erastin can cause both autophagy/lysosome-dependent degradation of ferritin and transcriptional upregulation of ferritin [40]. On the other hand, DAT-induced lysosomal degradation of ferritin in both autophagy-competent and autophagydefective MEFs, and the effect of DAT on ferritin was not associated with lysosomal degradation of Ncoa4 (Fig. 4c), indicating that DAT can induce the lysosomal degradation of ferritin, increase cellular free iron concentration, and thus sensitize cells to ferroptosis, in an autophagy and Ncoa4-independent manner.

In addition to autophagy (i.e., macroautophagy), chaperone-mediated autophagy (CMA) is another lysosome-mediated protein degradation pathway. In this process, a selective group of proteins containing a CMAtargeting motif are recognized by the chaperone protein
Hsc70 and subsequently delivered to the lysosomal surface to interact with LAMP2A, a lysosomal transmembrane protein, and the CMA cargo receptor. Through a series of complicated processes including complex assembly and disassembly, substrate proteins are translocated into the lysosome and degraded with LAMP2A [47]. To test whether CMA contributes to DAT-induced ferritin degradation, we analyzed the protein level of LAMP2A, and found that DAT did not induce LAMP2A degradation. Therefore, CMA is not involved in DAT-induced lysosomal degradation of ferritin (Fig. S7).

\section{DAT regulates cellular iron homeostasis via IRP-IRE signaling}

Iron homeostasis is under exquisite regulation in cells [33]. Iron regulatory protein-1 (IRP1) and IRP2 can sense cellular labile iron concentration. When cellular labile iron is low, IRP1 and IRP2 can bind to a cohort of mRNA molecules containing iron-responsive element (IRE) sequences, and thus dictating the stability or translational activity of these mRNAs (e.g., transferrin receptor mRNA is stabilized, and thus its protein synthesis is enhanced, whereas the translational activity of ferritin mRNA is suppressed). Since many of the proteins encoded by these mRNAs are involved in the regulation of cellular iron levels, the eventual consequence is that cells react by increasing cellular free iron concentration. Conversely, when cellular free iron is in excess, IRP1 will be associated with an iron-sulfur cluster and lose its affinity to IRE sequences, and IRP2 will be removed by proteasomal degradation, leading to a coordinated process to reduce cellular free iron. We sought to examine whether DAT can regulate cellular iron homeostasis via the IRP-IRE signaling.

To quantitatively measure the potential impact of DAT and erastin on the IRP-IRE signaling, IRE/IRP interaction and translational regulation of ferritin by erastin or DAT, two reporter plasmids, both based on the IRE sequence on the 5'-UTR region of ferritin mRNA, were used [37]. As shown in Fig. 5a, both plasmids express GFP as a reference through an internal ribosomal entry sequence (IRES), with one plasmid using luciferase as the readout (5'IRE-LucIRES-GFP) and the other using mCherry as the readout ( $5^{\prime}$ IRE-mCherry-IRES-GFP). These reporter systems can monitor IRP-IRE signaling: when cellular iron is low, IRPIRE association is enhanced, leading to a decrease of translation of luciferase or mCherry, thus a decreased ratio of luciferase/GFP or mCherry/GFP; conversely, when cellular iron is high, the ratio of luciferase/GFP or mCherry/ GFP will increase. As shown in Fig. 5b, in MEFs stably harboring the luciferase reporter, DAT treatment decreased the luciferase/GFP ratio, whereas erastin treatment increased the Luc/GFP ratio; consistently, in MEFs stably 
A

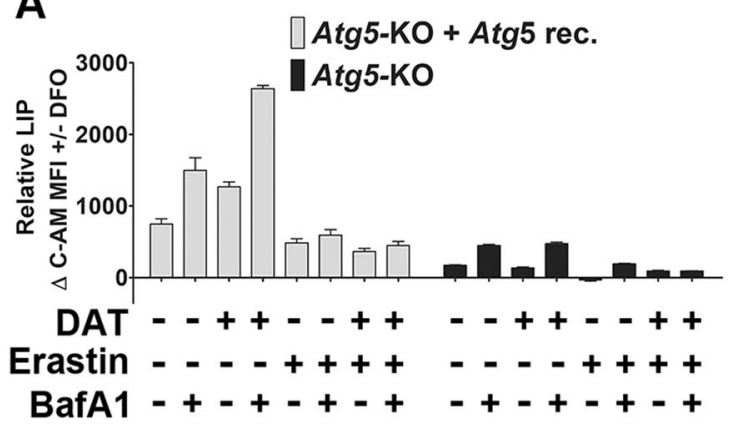

B

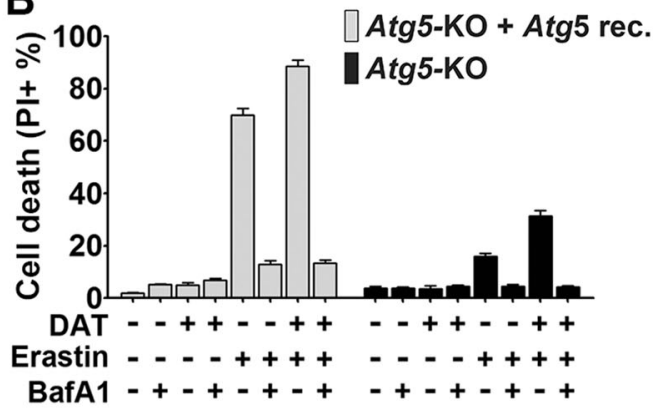

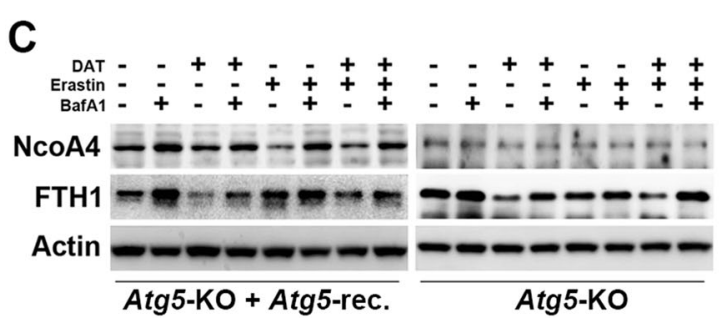

Fig. 4 Lysosomal activity is required for DAT-sensitized ferroptosis independent of autophagy. a Cellular LIP levels were quantified after treatment with erastin, DAT and/or BafA1 using the calcein-AM method by measuring spectrophotometric absorbance at $525 \mathrm{~nm}$. The MFI of C-AM is subtracted from the MFI of C-AM treated with DFO. b Percentage cell death was quantified by flow cytometric analysis of $\mathrm{PI}$-positive cells in autophagy-deficient Atg5-KO and autophagy- competent Atg5-reconstituted MEF cells after treatment with erastin, DAT and/or BafA1. c DAT can induce the lysosomal degradation of ferritin and increase cellular free iron. Western blot of NcoA4 and ferritin (FTH1) protein expression in Atg5-KO and Atg5-reconstituted MEFs treated with DAT, erastin or BafA1 for $6 \mathrm{~h}$. For $\mathbf{a}-\mathbf{c}$, cells were treated with $10 \mu \mathrm{M}$ DAT, $1 \mu \mathrm{M}$ erastin or $20 \mathrm{nM}$ BafA1

ascorbate $(500 \mu \mathrm{M})$ (Fig. $5 \mathrm{~d})$. As shown in Fig. 5e, the addition of $10 \mu \mathrm{M}$ of DAT, although sufficient to complex with all ferrous ion in the reaction system, did not inhibit lipid peroxidation. Therefore, DAT-associated iron maintains the ability to catalyze superoxidative reactions as free ferrous ion does.

\section{DAT sensitizes cancer cells to ferroptosis - therapeutic potential}

Ferroptosis induction has been explored as a new cancer therapeutic approach. For example, certain cancer cells, including mesenchymal cancer cells, are highly susceptible to GPX4 inhibition [13]. These findings suggest that GPX4 might be a promising therapeutic target for these metastasisprone cancer cells, which are often resistant to chemotherapy. On the other hand, many types of cancer cells are resistant to GPX4 inhibition. Given that DAT can sensitize cells to ferroptosis induced by GPX4 inhibitor RSL3 (Fig. S2), we set to test whether DAT can make these otherwise ferroptosis-resistant cancer cells more responsive to GPX4 inhibition. For this purpose, a cohort of human epithelial cancer cell lines, including human lung cancer cell H292, human colorectal cancer cells SW480, HCT116 and HT29 and human breast cancer cells MDA453 and MCF-7, were treated with different concentrations of RSL3. Compared with MEFs and HT1080 cells, which are sensitive to 
A

\begin{tabular}{l|l|l|l|l|l|} 
pBIG- CMV & IRE & LUC & IRES & GFP \\
\hline
\end{tabular}
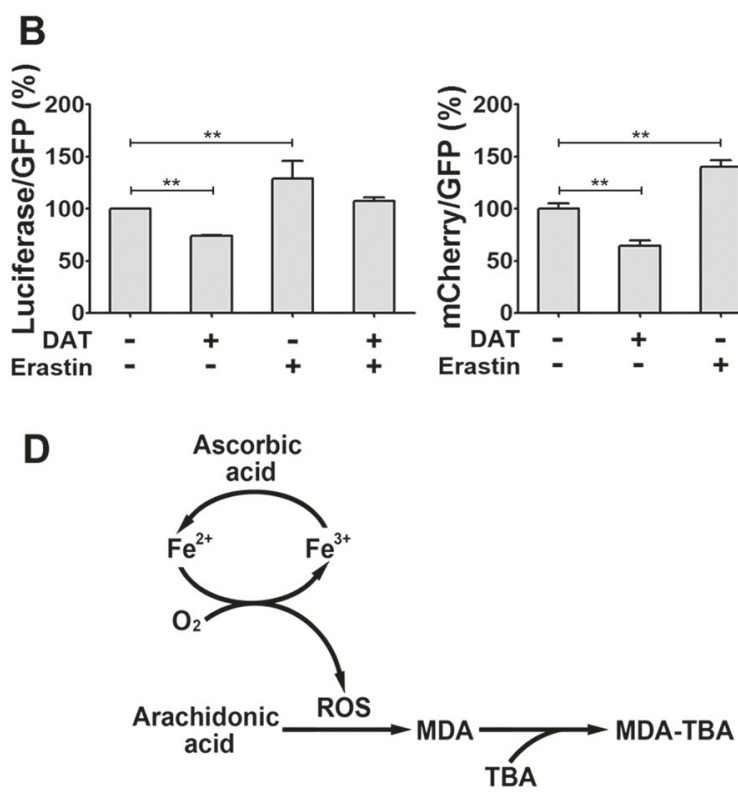

Fig. 5 DAT regulates cellular iron homeostasis via IRP-IRE signaling. a The structure of IRE-driven fluorescent reporters based on ferritin. b Quantitative evaluation of percentage luciferase/GFP and mCherry/ GFP regulated by the combination of DAT and erastin in MEF cells for $6 \mathrm{~h}$. Values are expressed as a percentage change from untreated controls. c Fluorescence microscopy images of mCherry and GFP in

RSL3, much lower ferroptosis and lipid peroxidation were induced in these cancer cells even with the highest concentration of RSL in this test (Fig. 6a). Importantly, DAT substantially increased the sensitivity of these cells to RSL3-induced cell death and lipid peroxidation in a ferrostatin-sensitive manner (Fig. 6b, c).

To further investigate this clinically relevant scenario, we performed in vivo mouse modeling experiments. Human H292 lung cancer cells, which are resistant to GPX4 inhibition (Fig. 6a), were engineered to harbor CRISPR/Cas9mediated GPX4 knockout in a Dox-inducible manner. Western blot analysis confirmed the efficiency of GPX4 knockout in H292 cells after Dox treatment (Fig. 6d). As expected, there was a low level of cell death and lipid ROS generation when GPX4 was knocked out, and both of which were enhanced by DAT in a ferrostatin-sensitive manner (Fig. 6d). Subsequently, we performed an in vivo xenograft experiment by subcutaneously inoculating nude mice with H292 cells harboring inducible GPX4 knockout. As shown in Fig. 6e-g, GPX4 expression is significantly decreased in tumors from mice fed with Dox diet. Tumor growth decreased modestly in mice treated with Dox diet or DAT alone, in comparison with control mice (-Dox-DAT).

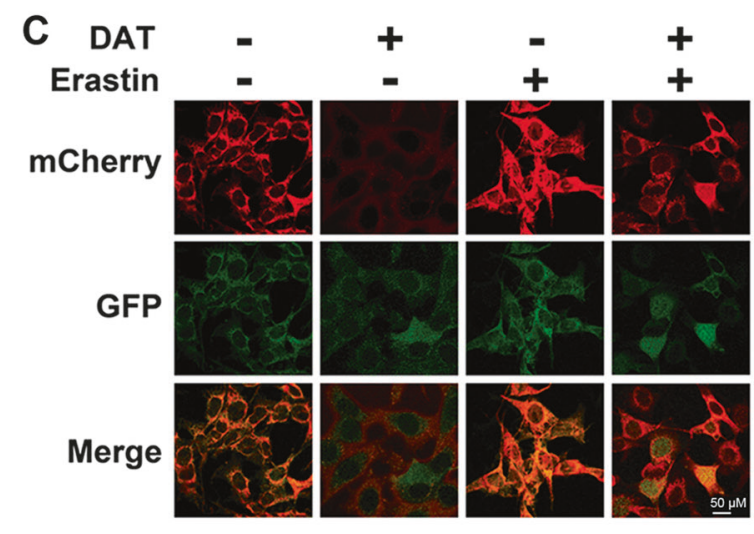

E

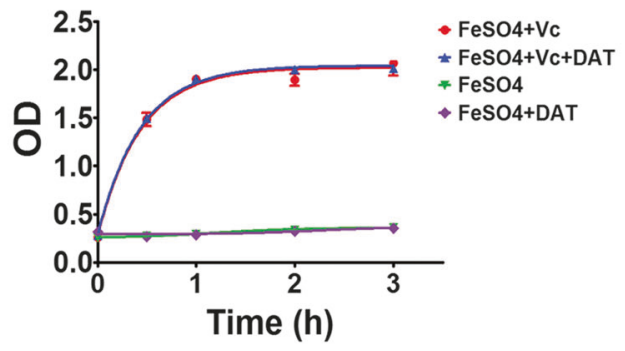

MEFs cells expressed with the mCherry/GFP reporter for $6 \mathrm{~h}$ and treated with DAT and/or erastin. $\mathbf{d}$ Schematic showing the oxidation of arachidonic acid catalyzed by ferrous ion and ascorbate. e DAT does not affect lipid peroxidation in the ferrous-ascorbate catalytic system. Plot shows the quantification of the oxidation of arachidonic acid to malondialdehyde

Importantly, in mice treated with the combination of Dox diet and DAT, tumor growth was further retarded. A significant decrease of Ki67 expression, indicative of cell proliferation, was also observed under this condition (Fig. 6g). Notably, Liproxstatin-1, a ferroptosis inhibitor amenable for in vivo use [49], partially restored tumor growth under this condition. Collectively, these results indicate that DAT can be used to improve the effect of GPX4-targeted therapy, especially for cancers that are more resistant to GPX4 inhibition.

\section{Discussion}

As highly effective antimalarial drugs, artemisinin and its derivatives have been explored for the treatment of various other diseases, particularly cancer [22, 23]. While positive outcomes have been reported in various experimental models for their effect in inhibiting cancer cell proliferation and inducing cancer cell death, the underlying molecular basis is far from clear. Further, in most studies, the anticancer effect of artemisinin compounds would not be apparent until extremely high concentrations (often $>100 \mu \mathrm{M}$ ) were used, 
A
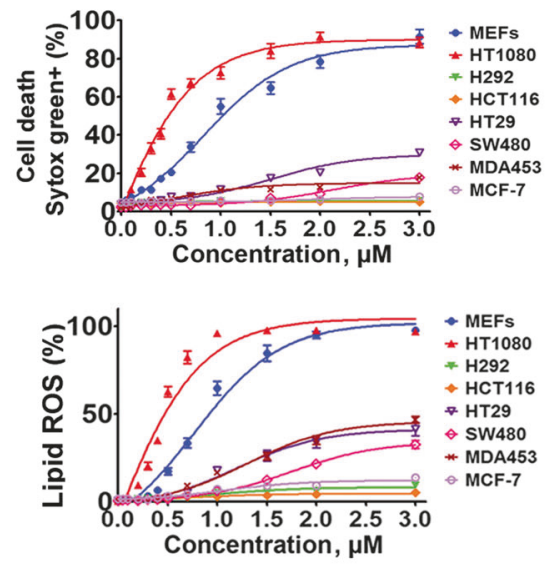

C
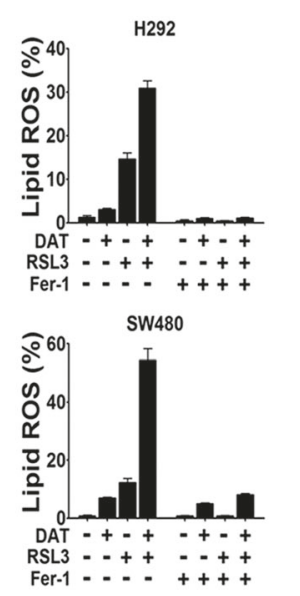

E

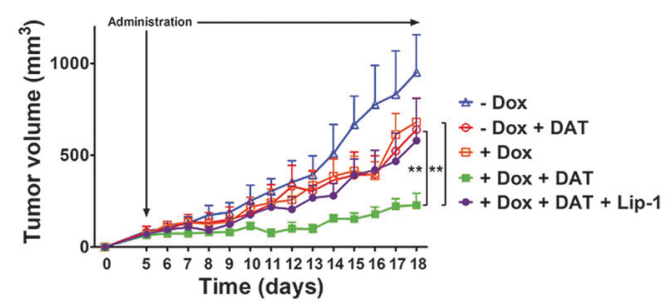

$\mathbf{F}_{\text {.Dox }}$
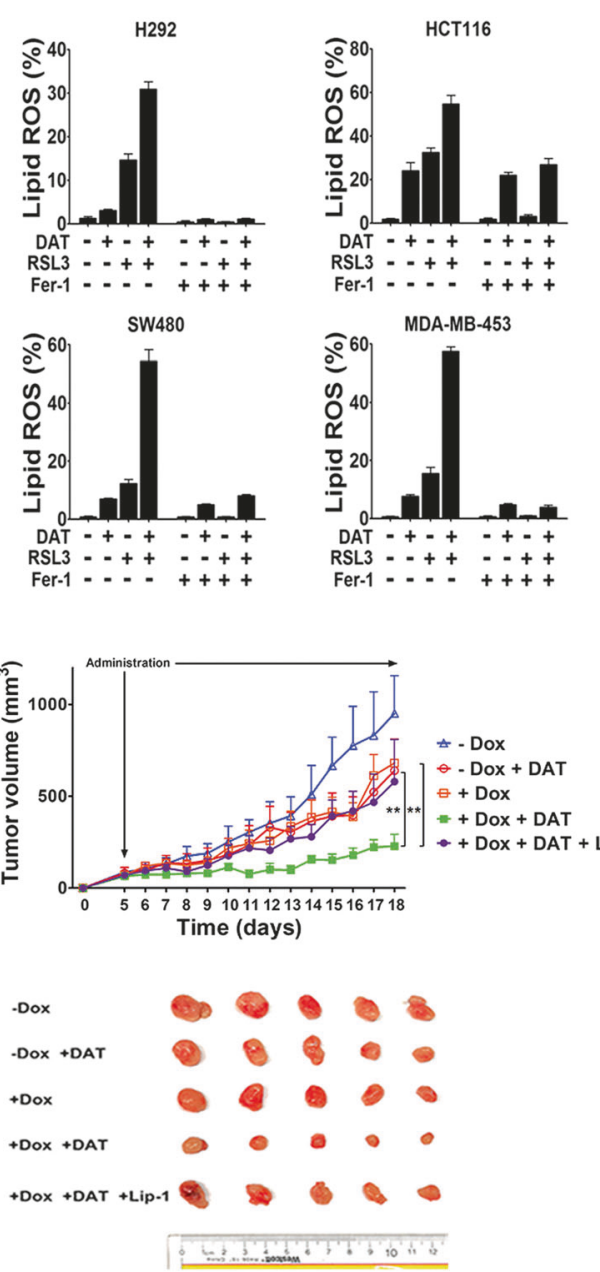

B

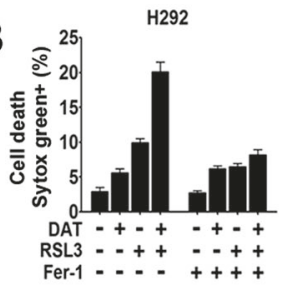

SW480

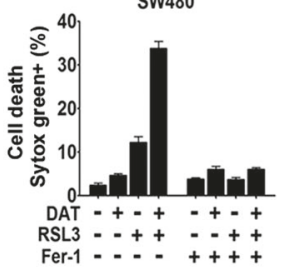

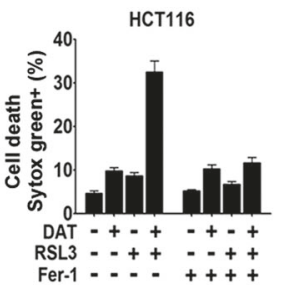

MDA-MB-453

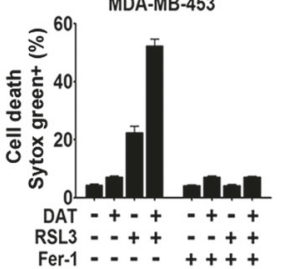

HT29
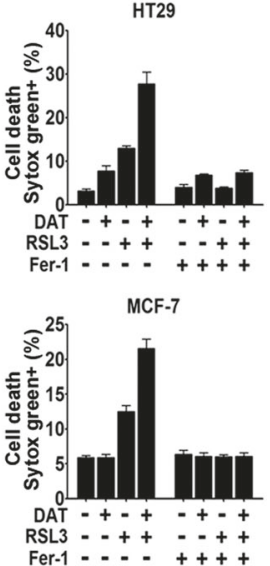
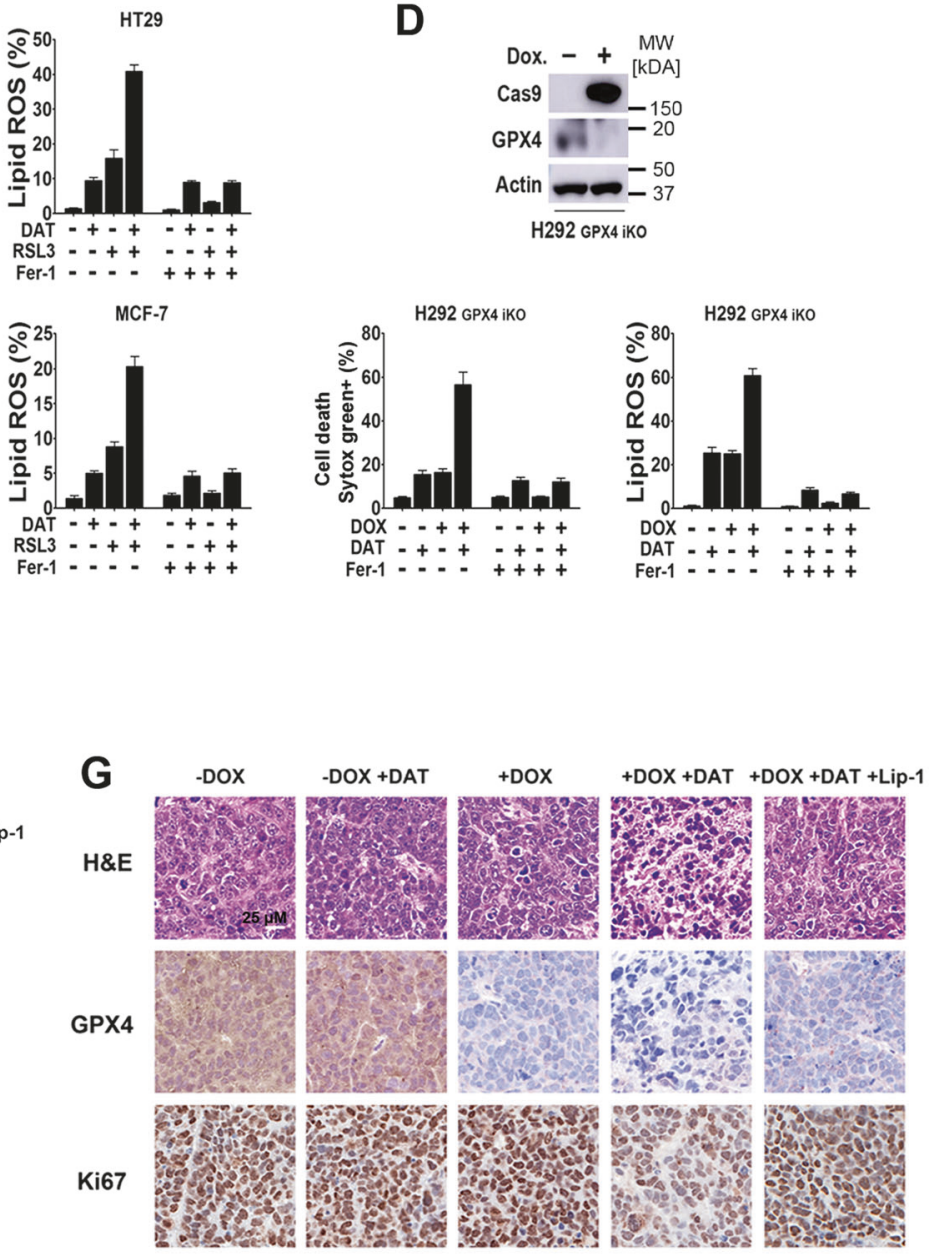

dampening their potential value in cancer treatment. Notably, even the precise mechanism of these compounds as antimalarial agents is still under intense debate. Numerous cellular proteins have been shown to interact with these compounds covalently or non-covalently [50-52]. However, it has not been defined whether the effects of artemisinin compounds are mediated explicitly by one or several of these interacting proteins. Albeit the ambiguity, it is generally believed that the peroxidative nature of the artemisinin compounds and their reactivity with cellular iron are essential for their function in killing both malaria and cancer cells [20,53].

In this study, we investigated the potential effect of artemisinin compounds in ferroptosis, by mainly focusing 
4 Fig. 6 DAT increases lipid ROS and sensitizes cancer cells to ferroptosis in vitro and in vivo. a Increasing concentrations of RSL3 were used to treat a panel of lung, breast and colorectal epithelial cancer cell lines. Cell death curves were plotted from percentage PI-positive cells quantified by flow cytometry. c Lipid ROS was uantified using the BODIPY-C11 lipid probe by flow cytometry after cells were treated with $2 \mu \mathrm{M}$ of RSL3 for $24 \mathrm{~h}$, or RSL3 with $1 \mu \mathrm{M}$ of ferroptosis inhibitor Ferrostatin-1 for $24 \mathrm{~h}$. d GPX4-iKO was induced using CRISPR/ doxycycline-inducible Cas9. Knockout was confirmed by western blot. e-g DAT sensitizes $\mathrm{H} 292$ cells to GPX4 KO induced ferroptosis in vivo. In e, Six- to eight-week old nude mice were inoculated with $3 \times 10^{6}$ doxycycline-inducible GPX4 iKO cells and randomly divided into 5 groups: vehicle (injected with PBS only), DAT only, doxycycline only, DAT and doxycycline and DAT, doxycycline and Liproxstatin-1. Tumor volume was measured daily using a Vernier caliper. Error bars represent standard error. ** Between - DAT + Dox and + Dox + DAT groups is $P=0.0049$. ** Between - Dox + DAT and + Dox + DAT groups is $P=0.0044$. $P$-values were calculated using a two-tailed $t$-test with $95 \%$ confidence interval. In f, the xenografted tumors were dissected and their size was measured as shown. In $\mathbf{g}$, xenografted tumors were sectioned, fixed and stained with H\&E, or immunohistochemically stained for GPX4 and proliferation marker Ki67

on DAT, the semi-synthetic derivative of artemisinin with the highest bioavailability and highest antimalarial activity [15]. Our study is based on the crucial role of peroxidation and iron in ferroptosis, and the chemical nature of artemisinin compounds as iron-reactive peroxides. Importantly, we found that by using DAT doses that are tolerable in vivo $[54,55]$, DAT sensitized cells to ferroptosis induced by both cysteine deprivation and GPX4 inhibition, although DAT failed to trigger ferroptosis by itself in our cell culture analysis (Fig. 1). Mechanistically, our results indicate that DAT associates with cellular free iron in such a way that DAT-associated iron still possesses the same oxidative activity as free iron, but it cannot be recognized by the IRPIRE iron homeostasis regulatory machinery. As a consequence, cells respond by enhancing lysosome-mediated ferritin degradation and IRP-mediated translational suppression of ferritin, both leading to an increase in cellular free iron contents to sensitize ferroptosis (Fig. 3-5). Related to our study, it was previously reported that artesunate, also an artemisinin derivative, can increase cellular free iron concentration by stimulating lysosomal degradation of ferritin, leading to apoptosis [56]. More recently, it was reported that 1,2-dioxolane $\mathrm{FINO}_{2}$, which contains endoperoxide like artemisinin compounds but is otherwise structurally distinctive, can initiate ferroptosis via iron oxidation and indirect inhibition of GPX4 [30].

A previous study showed that higher concentrations of ART $(50 \mu \mathrm{M})$ can induce cell death that can be rescued by necroptosis inhibitor Nec-1 [57]. Necroptosis and ferroptosis are two biochemically distinct forms of non-apoptotic cell death; although Nec-1 inhibits necroptosis by targeting RIP1, the compound has also been shown to protect against ferroptosis in a RIP1-independent manner [49]. Therefore, the Nec-1 experiment alone is insufficient for determining whether cells undergo necroptosis. Another significant difference between this previous study and ours is that the previous study used a high dose of ART as a single cell death-inducing agent, whereas here we used a low dose of DAT to sensitize ferroptosis triggered by multiple types of inducers.

Interestingly, both DAT and the ferroptosis inducer erastin can increase cellular free/labile iron, but via a somewhat different mechanism. Erastin can stimulate autophagic degradation of ferritin (ferritinophagy [58]) and thus an increase of cellular labile iron [40]. The increased labile iron engages the IRP-IRE iron homeostatic system, leading to the upregulation of ferritin synthesis to mitigate further increase of cellular labile iron [33]. In stark contrast to erastin, DAT-induced lysosomal degradation of ferritin and the increase of cellular labile iron are independent of autophagy (Fig. 4a, c). Further, DAT can associate with and "mask" free iron, thus evading the feedback regulation by the IRP-IRE system to cause a continued increase of cellular free iron (Fig. 5b, c, e).

Importantly, our finding that DAT can sensitize ferroptosis has a clear cancer therapeutic implication. While the use of ferroptosis-inducing agents is an attractive novel therapeutic option for some types of cancers, it is important to note that there are currently certain challenges associated with the preclinical and clinical use of these agents. Whereas DAT is generally well-tolerated, ferroptosis-inducing compounds may lead to tissue damage by stimulating lipid peroxidation and ferroptotic death. Thus, long-term use of these compounds can potentially cause or exacerbate iron metabolic diseases such as diabetes [59], atherosclerosis [60], neuronal diseases [61] and myocardial dysfunction [62]. Albeit these potential side effects, ferroptosis induction is still considered a promising cancer therapeutic approach, especially for cancer cells that are highly sensitive to ferroptosis. Indeed, a plethora of mesenchymal cancer cells that are prone to metastasis and are often resistant to chemotherapies and various targeted therapies, are highly susceptible to ferroptosis induction [13]. Therefore, a relatively low dose of ferroptosis inducers, hopefully tolerable by normal tissues, may be effective for the treatment of these cancers. Using DAT to further sensitize ferroptosis could shorten the treatment time and improve the therapeutic effect.

Acknowledgements The authors thank members of the Jiang and Bian labs for advice, technical assistance and for critically reading the manuscript. This work is supported by NIH grants R01CA204232 \& R01GM113013 (X.J.), Geoffrey Beene Cancer Research fund (X.J.), NCI Cancer Center Core Grant P30CA008748 for Memorial Sloan-Kettering Cancer Center, China Scholarship Council Fellowship 201603170375 (J.W.), and HKRGC GRF grant 12104415 (Z-X.B.). 


\section{Compliance with ethical standards}

Conflict of interest The authors declare that they have no conflict of interest.

Publisher's note: Springer Nature remains neutral with regard to jurisdictional claims in published maps and institutional affiliations.

\section{References}

1. Elmore S. Apoptosis: a review of programmed cell death. Toxicol Pathol. 2007;35:495-516.

2. Ashkenazi A, Salvesen G. Regulated cell death: signaling and mechanisms. Annu Rev Cell Dev Biol. 2014;30:337-56.

3. Galluzzi L, Vitale I, Aaronson SA, Abrams JM, Adam D, Agostinis P, et al. Molecular mechanisms of cell death: recommendations of the Nomenclature Committee on Cell Death 2018. Cell Death Differ. 2018;25:486-541.

4. Dixon SJ, Lemberg KM, Lamprecht MR, Skouta R, Zaitsev EM, Gleason CE, et al. Ferroptosis: an iron-dependent form of nonapoptotic cell death. Cell. 2012;149:1060-72.

5. Gao M, Monian P, Quadri N, Ramasamy R, Jiang XJ. Glutaminolysis and transferrin regulate ferroptosis. Mol Cell. 2015;59:298-308.

6. Stockwell BR, Friedmann Angeli JP, Bayir H, Bush AI, Conrad M, Dixon SJ, et al. Ferroptosis: a regulated cell death nexus linking metabolism, redox biology, and disease. Cell. 2017;171:273-85.

7. Conrad M, Angeli JP, Vandenabeele P, Stockwell BR. Regulated necrosis: disease relevance and therapeutic opportunities. Nat Rev Drug Discov. 2016;15:348-66.

8. Toyokuni S, Ito F, Yamashita K, Okazaki Y, Akatsuka S. Iron and thiol redox signaling in cancer: An exquisite balance to escape ferroptosis. Free Radic Biol Med. 2017;108:610-26.

9. Tonnus W, Linkermann A. The in vivo evidence for regulated necrosis. Immunol Rev. 2017;277:128-49.

10. Wang SJ, Li D, Ou Y, Jiang L, Chen Y, Zhao Y, et al. Acetylation Is Crucial for p53-Mediated Ferroptosis and Tumor Suppression. Cell Rep. 2016;17:366-73.

11. Zhang Y, Shi J, Liu X, Feng L, Gong Z, Koppula P, et al. BAP1 links metabolic regulation of ferroptosis to tumour suppression. Nat Cell Biol. 2018;20:1181-92.

12. Gao M, Yi J, Zhu J, Minikes AM, Monian P, Thompson CB, et al. Role of mitochondria in ferroptosis. Mol Cell. 2018;72:354-63.

13. Viswanathan VS, Ryan MJ, Dhruv HD, Gill S, Eichhoff OM, Seashore-Ludlow B, et al. Dependency of a therapy-resistant state of cancer cells on a lipid peroxidase pathway. Nature. 2017;547:453-7.

14. Klayman DL. Qinghaosu (artemisinin): an antimalarial drug from China. Science. 1985;228:1049-55.

15. Balint GA. Artemisinin and its derivatives: an important new class of antimalarial agents. Pharm Ther. 2001;90:261-5.

16. O'Neill PM, Barton VE, Ward SA. The molecular mechanism of action of artemisinin-the debate continues. Molecules. 2010;15:1705-21.

17. Cui L, Su XZ. Discovery, mechanisms of action and combination therapy of artemisinin. Expert Rev Anti Infect Ther. 2009;7:999-1013.

18. Krishna S, Uhlemann AC, Haynes RK. Artemisinins: mechanisms of action and potential for resistance. Drug Resist Updat. 2004; 7:233-44.

19. Asawamahasakda W, Ittarat I, Pu YM, Ziffer H, Meshnick SR. Reaction of antimalarial endoperoxides with specific parasite proteins. Antimicrob Agents Chemother. 1994;38:1854-8.
20. Meshnick SR. The mode of action of antimalarial endoperoxides. Trans R Soc Trop Med Hyg. 1994;88(Suppl 1):S31-2.

21. Zhang F, Gosser DK Jr., Meshnick SR. Hemin-catalyzed decomposition of artemisinin (qinghaosu). Biochem Pharmacol. 1992;43:1805-9.

22. Efferth T. Molecular pharmacology and pharmacogenomics of artemisinin and its derivatives in cancer cells. Curr Drug Targets. 2006;7:407-21.

23. Chaturvedi D, Goswami A, Saikia PP, Barua NC, Rao PG. Artemisinin and its derivatives: a novel class of anti-malarial and anti-cancer agents. Chem Soc Rev. 2010;39:435-54.

24. Konstat-Korzenny E, Ascencio-Aragon JA, Niezen-Lugo S, Vazquez-Lopez R. Artemisinin and its synthetic derivatives as a possible therapy for cancer. Med Sci. 2018;6:19.

25. Berdelle N, Nikolova T, Quiros S, Efferth T, Kaina B. Artesunate induces oxidative DNA damage, sustained DNA double-strand breaks, and the ATM/ATR damage response in cancer cells. Mol Cancer Ther. 2011;10:2224-33.

26. Li PC, Lam E, Roos WP, Zdzienicka MZ, Kaina B, Efferth T. Artesunate derived from traditional Chinese medicine induces DNA damage and repair. Cancer Res. 2008;68:4347-51.

27. Wang Z, Hu W, Zhang JL, Wu XH, Zhou HJ. Dihydroartemisinin induces autophagy and inhibits the growth of iron-loaded human myeloid leukemia K562 cells via ROS toxicity. FEBS Open Bio. 2012;2:103-12.

28. Singh NP, Lai HC. Artemisinin induces apoptosis in human cancer cells. Anticancer Res. 2004;24:2277-80.

29. Ooko E, Saeed ME, Kadioglu O, Sarvi S, Colak M, Elmasaoudi $\mathrm{K}$, et al. Artemisinin derivatives induce iron-dependent cell death (ferroptosis) in tumor cells. Phytomedicine. 2015;22:1045-54.

30. Gaschler MM, Andia AA, Liu H, Csuka JM, Hurlocker B, Vaiana $\mathrm{CA}$, et al. FINO2 initiates ferroptosis through GPX4 inactivation and iron oxidation. Nat Chem Biol. 2018;14:507-15.

31. Chen G, Gong R, Shi X, Yang D, Zhang G, Lu A, et al. Halofuginone and artemisinin synergistically arrest cancer cells at the G1/G0 phase by upregulating p21Cip1 and p27Kip1. Oncotarget. 2016;7:50302-14.

32. Chen T, Li M, Zhang R, Wang H. Dihydroartemisinin induces apoptosis and sensitizes human ovarian cancer cells to carboplatin therapy. J Cell Mol Med. 2009;13:1358-70.

33. Rouault TA. The role of iron regulatory proteins in mammalian iron homeostasis and disease. Nat Chem Biol. 2006;2:406-14.

34. Prus E, Fibach E. Flow cytometry measurement of the labile iron pool in human hematopoietic cells. Cytom A. 2008;73:22-7.

35. Esterbauer H, Cheeseman KH. Determination of aldehydic lipid peroxidation products: malonaldehyde and 4-hydroxynonenal. Methods Enzym. 1990;186:407-21.

36. De la Cruz JP, Olveira C, Gonzalez-Correa JA, Benitez A, Sanchez, de la Cuesta F. Inhibition of ferrous-induced lipid peroxidation by dipyridamole, RA-642 and mopidamol in human lung tissue. Gen Pharmacol. 1996;27:855-9.

37. Li JY, Ram G, Gast K, Chen X, Barasch K, Mori K, et al. Detection of intracellular iron by its regulatory effect. Am J Physiol Cell Physiol. 2004;287:C1547-59.

38. Yang WS, SriRamaratnam R, Welsch ME, Shimada K, Skouta R, Viswanathan VS, et al. Regulation of ferroptotic cancer cell death by GPX4. Cell. 2014;156:317-31.

39. Hou W, Xie Y, Song X, Sun X, Lotze MT, Zeh HJ 3rd, et al. Autophagy promotes ferroptosis by degradation of ferritin. Autophagy. 2016;12:1425-8.

40. Gao M, Monian P, Pan Q, Zhang W, Xiang J, Jiang X. Ferroptosis is an autophagic cell death process. Cell Res. 2016;26:1021-32.

41. Zhang Z, Yao Z, Zhao S, Shao J, Chen A, Zhang F, et al. Interaction between autophagy and senescence is required for dihydroartemisinin to alleviate liver fibrosis. Cell Death Dis. 2017;8: e2886. 
42. Wang J, Pantopoulos K. Regulation of cellular iron metabolism. Biochem J. 2011;434:365-81.

43. Asano T, Komatsu M, Yamaguchi-Iwai Y, Ishikawa F, Mizushima N, Iwai K. Distinct mechanisms of ferritin delivery to lysosomes in iron-depleted and iron-replete cells. Mol Cell Biol. 2011;31:2040-52.

44. Kurz T, Eaton JW, Brunk UT. The role of lysosomes in iron metabolism and recycling. Int J Biochem. Cell B. 2011;43:1686-97.

45. Radisky DC, Kaplan J. Iron in cytosolic ferritin can be recycled through lysosomal degradation in human fibroblasts. Biochem $\mathrm{J}$. 1998;336:201-5.

46. Mancias JD, Wang X, Gygi SP, Harper JW, Kimmelman AC. Quantitative proteomics identifies NCOA4 as the cargo receptor mediating ferritinophagy. Nature. 2014;509:105-9.

47. Dice JF. Chaperone-mediated autophagy. Autophagy. 2007;3: 295-9.

48. Sibmooh N, Udomsangpetch R, Kujoa A, Chantharaksri U, Mankhetkorn S. Redox reaction of artemisinin with ferrous and ferric ions in aqueous buffer. Chem Pharm Bull. 2001;49:1541-6.

49. Friedmann Angeli JP, Schneider M, Proneth B, Tyurina YY, Tyurin VA, Hammond VJ, et al. Inactivation of the ferroptosis regulator Gpx4 triggers acute renal failure in mice. Nat Cell Biol. 2014;16:1180-91.

50. Eckstein-Ludwig U, Webb RJ, Van Goethem ID, East JM, Lee AG, Kimura M, et al. Artemisinins target the SERCA of Plasmodium falciparum. Nature. 2003;424:957-61.

51. Eichhorn T, Winter D, Buchele B, Dirdjaja N, Frank M, Lehmann WD, et al. Molecular interaction of artemisinin with translationally controlled tumor protein (TCTP) of Plasmodium falciparum. Biochem Pharm. 2013;85:38-45.

52. Willoughby JA, Sundar SN, Cheung M, Tin AS, Modiano J, Firestone GL. Artemisinin blocks prostate cancer growth and cell cycle progression by disrupting $\mathrm{Sp} 1$ interactions with the cyclindependent kinase-4 (CDK4) promoter and inhibiting CDK4 gene expression. J Biol Chem. 2009;284:2203-13.
53. Hong YL, Yang YZ, Meshnick SR. The interaction of artemisinin with malarial hemozoin. Mol Biochem Parasitol. 1994;63:121-8.

54. Wang SJ, Gao Y, Chen H, Kong R, Jiang HC, Pan SH, et al. Dihydroartemisinin inactivates NF-kappaB and potentiates the anti-tumor effect of gemcitabine on pancreatic cancer both in vitro and in vivo. Cancer Lett. 2010;293:99-108.

55. Chen H, Sun B, Pan S, Jiang H, Sun X. Dihydroartemisinin inhibits growth of pancreatic cancer cells in vitro and in vivo. Anticancer Drugs. 2009;20:131-40.

56. Yang ND, Tan SH, Ng S, Shi Y, Zhou J, Tan KS, et al. Artesunate induces cell death in human cancer cells via enhancing lysosomal function and lysosomal degradation of ferritin. J Biol Chem. 2014;289:33425-41.

57. Button RW, Lin F, Ercolano E, Vincent JH, Hu B, Hanemann CO, et al. Artesunate induces necrotic cell death in schwannoma cells. Cell Death Dis. 2014;5:e1466.

58. Dowdle WE, Nyfeler B, Nagel J, Elling RA, Liu S, Triantafellow E, et al. Selective VPS34 inhibitor blocks autophagy and uncovers a role for NCOA4 in ferritin degradation and iron homeostasis in vivo. Nat Cell Biol. 2014;16:1069-79.

59. Huang J, Jones D, Luo B, Sanderson M, Soto J, Abel ED, et al. Iron overload and diabetes risk: a shift from glucose to Fatty Acid oxidation and increased hepatic glucose production in a mouse model of hereditary hemochromatosis. Diabetes. 2011;60:80-7.

60. Dabbagh AJ, Shwaery GT, Keaney JF Jr., Frei B. Effect of iron overload and iron deficiency on atherosclerosis in the hypercholesterolemic rabbit. Arterioscler Thromb Vasc Biol. 1997; 17:2638-45.

61. Hare D, Ayton S, Bush A, Lei P. A delicate balance: Iron metabolism and diseases of the brain. Front Aging Neurosci. 2013;5:34.

62. Gammella E, Recalcati S, Rybinska I, Buratti P, Cairo G. Ironinduced damage in cardiomyopathy: oxidative-dependent and independent mechanisms. Oxid Med Cell Longev. 2015; 2015:230182. 